\title{
Comunicación e igualdad en una sociedad compleja
}

\author{
Cristian CABALÍN QUIJADA*
}

\section{Introducción}

En el contexto de la globalización, la desigualdad entre Estados, economías y personas es una de las mayores preocupaciones. No se trata sólo de una disparidad material en cuanto al acceso a bienes, sino que además contempla injusticia social, precariedad del trabajo, problemas medioambientales, deslegitimación de la democracia, entre otros grandes conflictos. Tal como asegura Ulrick Beck, "la producción social de la riqueza va acompañada sistemáticamente por la producción de riesgos". . En este sentido, uno de los principales riesgos de la democracia y del Estado de Derecho es la desigualdad en la distribución de la riqueza, que puede provocar estallidos sociales producto del descontento generalizado de la población. Chile no escapa a esta realidad y presenta un grave conflicto de redistribución de los ingresos, que constituye el indicador fundamental en materia de equidad. Por ejemplo, la distancia entre el $10 \%$ más rico y el $10 \%$ más pobre fue de 34,33 veces el año 2003, superior a la registrada en el periodo 1990 y $1998^{2}$.

Durante la campaña presidencial de 2005, que llevó finalmente a Michelle Bachelet a La Moneda, la desigualdad fue uno de los principales temas de discusión. Todos los candidatos incorporaron en sus programas de gobiernos soluciones a la inequidad y entregaron sus propuestas en los debates televisivos y entrevistas. Así, se creó en la opinión pública una corriente preocupada de la inequidad social, entendiendo que "la opinión pública ya no comprende a todo el sistema social, sino al político como sistema parcial, mediante comunicaciones públicas derivadas de una selección contingente de temas"3. Esta definición de opinión pública tiene su base en la teoría sociopoiética de la sociedad esgrimida por el sociólogo alemán Niklas Luhmann. Para este pensador, la sociedad es un sistema cerrado, operacionalmente clausurado y diferencialmente funcional, constituido por la comunicación ${ }^{4}$.

Con base justamente en este programa de observación de la realidad y en la Teoría de la Acción Comunicativa de Jürgen Habermas, este artículo propone una intervención comunicacional en torno a la igualdad como una herramienta más para promover la disminución de las diferencias sociales. De acuerdo con Luhmann, la sociedad es comunicación, por lo tanto, es posible la construcción de la realidad social a partir de comunicaciones. De hecho, las comunicaciones desviantes constituyen el primer proceso en la evolución de las sociedades 5 . Además, considerando la importancia de los medios masivos de comunicación en la sociedad actual, un trabajo en este sentido tiene amplias posibilidades de materialización.

\footnotetext{
* Periodista y licenciado en Comunicación en Comunicación Social. Candidato a Magíster en Antropología y Desarrollo en la Universidad de Chile

${ }^{1}$ BECK, Ulrick. La sociedad del riesgo. Hacia una nueva modernidad. Ediciones Paidós, Barcelona. 1998.

${ }^{2}$ FAZIO, Hugo. Mapa de la extrema riqueza al año 2005. Lom Ediciones, Santiago de Chile. 2005.

${ }^{3}$ TORRES, Emilio y DE LA PUENTE, Patricio. Seguridad ciudadana y sistemas sociales autorreferentes en el contexto de la sociedad compleja. Revista MAD número 5. Departamento de Antropología de la Universidad de Chile. Santiago de Chile. 2001.

${ }^{4}$ LUHMANN, Niklas y DEGIORGI, Raffaele. Teoría de la Sociedad. Universidad de Guadalajara. Universidad Iberoamericana. Instituto Tecnológico y de Estudios Superiores de Occidente. México. 1993. ${ }^{5}$ Ibid.
} 
Es así como se establece una relación entre comunicación y desarrollo, donde se rescata principalmente la necesidad de generar un "ambiente" favorable al cambio social, que considere entre sus principales semánticas a la igualdad.

\section{Inequidad globalizada y la realidad chilena}

"La globalización supone una interacción funcional de actividades económicas y culturales dispersas, bienes y servicios generados por un sistema con muchos centros, en el quq importa más la velocidad para recorrer el mundo que las posiciones geográficas desde las cuales actúa"6. Ciertamente, la rapidez en las comunicaciones, transacciones y en el consumo es una de las principales características de la globalización, pero las posiciones geográficas desde donde actúa sí tienen importancia. Así lo demuestra la actual estructura de la economía mundial donde los países industrializados - unidos en el G8- no poseen el nivel de desigualdad y marginalidad que se presenta en los países del Tercer Mundo, principalmente en América Latina, África y en parte de Asia. Todos ellos están al sur, o sea, al parecer la posición geográfica cobra relevancia. Pero más allá de este problema de ubicación, lo cierto es que la gran mayoría de las naciones subdesarrolladas presenta serios problemas sociales, principalmente, expresados en enormes capas de pobreza y en grotescas disparidades en la distribución de la riqueza.

Desde la segunda mitad siglo XX, los países han declarado sus intenciones de erradicar la pobreza en todo el mundo. El programa Milenio de Naciones Unidas, de hecho, lo contempla entre sus principales objetivos. Sin embargo, en las últimas décadas, el número de pobres se ha incrementado en 100 millones de personas. Esto ha ocurrido al mismo tiempo que el crecimiento de la economía mundial aumenta en un promedio de 2,5 puntos porcentuales anuales ${ }^{7}$. Es decir, mientras crece el producto interno bruto de los países, sube la pobreza a nivel mundial.

La economía globalizada no ha sido capaz de satisfacer las necesidades básicas de la mayoría de los habitantes del planeta. De hecho, el desigual reparto de los bienes se ha convertido en un idéntico reparto de los males en las naciones más atrasadas. Sin embargo, los países pobres o con peores indicadores de Desarrollo Humano no son siempre los más injustos en la distribución de la riqueza.

Es el caso de Chile, que ha logrado reducir la pobreza en un $20 \%$, pese a que existen críticas a la metodología para medir la precariedad y al indicador que se utiliza para determinar si una persona es pobre o no. Con 43 mil pesos al mes, un habitante de Chile deja ser considerado pobre, cifra mezquina según un estudio de Fundación Terram, que situó un umbral de satisfacción mínima en 130 mil pesos ${ }^{8}$. Más allá de este legítimo cuestionamiento a la superación de la pobreza, Chile ha demostrado ciertos avances en la promoción del desarrollo, pero ha fallado en generar condiciones de igualdad en la distribución de la riqueza. De hecho, "posee una de las economías más desiguales del mundo. De acuerdo con el coeficiente Gini -en que un nivel cero indica una sociedad perfectamente igualitaria, mientras que un nivel cien indica una sociedad totalmente desigualitaria- Chile tiene un coeficiente 58, cercano a Brasil, caracterizado mundialmente

\footnotetext{
${ }^{6}$ GARCÍA CANCLINI, Néstor. Consumidores y ciudadanos. Conflictos multiculturales de la globalización. Grijalbo. México. 1995.

${ }^{7}$ STIGLITZ, Joseph. El malestar de la globalización. Editorial Santillana. Buenos Aires. 2002.

${ }^{8}$ CLAUDE, Marcel. Determinación del nuevo umbral para la pobreza en Chile. Fundación Terram. Santiago de Chile. 2001.
} 
por su mala distribución en el ingreso"9. El siguiente cuadro muestra el Índice Gini en 1981 y en 2001.

\section{Cuadro I: Distribución de los Ingresos, Índice Gini}

\begin{tabular}{|c|c|c|}
\hline País & $\mathbf{1 9 8 1}$ & $\mathbf{2 0 0 1}$ \\
\hline Brasil & 57,6 & 59,3 \\
\hline Chile & 56,4 & 57,3 \\
\hline China & 25,0 & 36,3 \\
\hline Ecuador & 43,9 & 53,4 \\
\hline India & 30,1 & 28,1 \\
\hline Malasia & 46,3 & 49,2 \\
\hline México & 39,2 & 54,9 \\
\hline Perú & 23,3 & 49,8 \\
\hline Sudáfrica & 60,7 & 57,8 \\
\hline Venezuela & 60,1 & 49,5 \\
\hline
\end{tabular}

Fuente: Banco Mundial, en porcentajes, por orden alfabético.

Tal como muestra el cuadro anterior, la injusta distribución de los ingresos es un problema mundial, que se manifiesta principalmente en los países del Tercer Mundo. En 20 años, Chile ha experimentado una situación regresiva que demuestra que las políticas empleadas para disminuir las desigualdades no son del todo exitosas. Por eso, no extraña que después de 16 años de transición a la democracia, recién en la última campaña presidencial fuera uno de los principales temas en la opinión pública, escoltando a la delincuencia que ocupó la mayor atención de los candidatos.

La delincuencia puede ser considerada justamente uno de los riesgos de la sociedad moderna, a los cuales hace mención Beck. La extrema desigualdad se vincula a un grave proceso de deterioro de la cohesión social, que puede tener consecuencias en la estabilidad del sistema democrático, de la economía y en la generación también de los movimientos de protestas, como pueden ser los focos delictivos en la sociedad moderna. La inequidad no sólo se expresa en la capacidad material y de consumo de las personas, sino también en las posibilidades de acceso al mercado del trabajo y a la educación. De hecho, según la encuesta Nacional Urbana de Seguridad Ciudadana del Ministerio del Interior de 2003, el $33,1 \%$ de los encuestados cree que la principal causa de los actuales niveles de delincuencia es la falta de trabajo.

Aunque el dato anterior sólo hace referencia a una percepción de los entrevistados para ese estudio, cifras del Instituto Nacional de Estadísticas (INE) ratifican alguna vinculación entre delincuencia y problemas sociales, ya que los delincuentes comienzan su actividad delictiva entre los 15 y 17 años y el $66 \%$ de ellos tiene un nivel escolar incompleto. Además, el desempleo en el trimestre móvil enero-marzo de 2006 llegó al $7,9 \%$ mientras que el desempleo juvenil (15 a 24 años) alcanzó el 17,2\%. Obviamente, no existe una relación lineal entre desigualdad-marginalidad-delincuencia, ya que el problema de la exclusión social es multifacético, pero sí se pueden establecer ciertos riesgos asociados a la desigualdad.

\footnotetext{
${ }^{9}$ FAZIO, Hugo. Op. Cit.
} 
"El cambio de la lógica del reparto de la riqueza en la sociedad de la carencia a la lógica del reparto de los riesgos en la modernidad desarrollada está vinculado históricamente a dos condiciones. En primer lugar, este cambio se consuma allí donde y en la medida en que mediante el nivel alcanzado por las fuerzas productivas humanas y tecnológicas y por las seguridades y regulaciones del Estado social se pueden reducir objetivamente, excluir socialmente la miseria material auténtica. En segundo lugar, este cambio categorial depende al mismo tiempo de que al hilo del crecimiento exponencial de las fuerzas productivas en el proceso de modernización se liberen los riesgos y los potenciales de auto amenaza en una medida desconocida hasta el momento" 10 .

Una de las amenazas para la sociedad y para su estabilidad institucional es la desigualdad. Por ello, es necesario articular una estrategia de intervención social y comunicacional que permita generar un ambiente de reconocimiento de la necesidad de vivir en una sociedad igualitaria. En Chile, esta semántica no se ha condensado y aún no se entiende que "el tema complemento al de la inclusión es el fenómeno de la exclusión y que no se trata en él simplemente de una marginación, de una falta de integración. Estos grupos pueden estar integrados, pero resultan invisibles para los subsistemas funcionales porque no cuentan con las condiciones mínimas para ser considerados"11.

\section{La comunicación en la sociedad compleja}

En la sociedad moderna de hoy, compleja y sociopoiética, la comunicación tiene un papel constitutivo de la sociedad. "La comunicación tiene todas las propiedades necesarias para la autopoiesis del sistema: es una operación genuinamente social (y la única genuinamente tal). Es una operación social, porque presupone el concurso de un gran número de sistemas de conciencia, pero precisamente por eso, como unidad, no puede ser imputada a ninguna conciencia sola. Es social porque de ningún modo puede ser producida una conciencia común colectiva, es decir, no se puede llegar al consenso en el sentido de un acuerdo completo; y sin embargo, la comunicación funciona" ${ }^{\prime 2}$. En otras palabras, los sistemas sociales están conformados por comunicación, entendida como el intercambio de códigos.

Pese a que Luhmann entiende la comunicación de una manera totalmente alejada a lo que plantea Jürgen Habermas, se pueden emplear ciertos conceptos de la teoría sociopoiética para una intervención comunicacional. Habermas ${ }^{13}$ plantea una racionalidad comunicativa, donde los actores sociales se relacionan orientándose hacia el entendimiento. Esta direccionalidad de la comunicación choca con los planteamientos de Luhmann, quien señala que la comunicación es altamente improbable y que no tiene una finalidad más que su autorreproducción. De todos modos, ambos pensadores enfatizan la importancia de la comunicación en la sociedad moderna y es ahí donde sus planteamientos tienen importancia en una estrategia de intervención comunicacional.

Con la globalización se han masificado aún más los medios de comunicación, ya no sólo la imprenta, la televisión o el cine están presentes, sino también Internet, la televisión digital y la multimedia. Existe un acelerado consumo de contenidos de los medios de

\footnotetext{
${ }^{10}$ BECK, Ulrick. Op. Cit.

${ }^{11}$ ARRIAGA, Emilio. La Teoría de Niklas Luhmann. Centro de Innovación, Desarrollo e Investigación Educativa. Universidad Autónoma del Estado de México. México. 2000.

${ }^{12}$ LUHMANN, N. y DEGIORGI, R. Op. Cit.

${ }^{13}$ HABERMAS, Jürgen. Teoría de la Acción Comunicativa. Editorial Taurus. Madrid. 1987.
} 
comunicación, donde se produce una vorágine de manifestaciones simbólicas y producción cultural $^{14}$. Esta incesante producción de comunicaciones está obviamente asociada a los medios masivos de comunicación.

Aunque sus efectos e influencia han estado en cuestionamiento en las últimas décadas, los medios masivos de comunicación son uno de los ejes relevantes de las comunicaciones y de la opinión pública en la sociedad moderna. Por ejemplo, una encuesta del Programa de Naciones Unidas para el Desarrollo Humano (PNUD) indicó que la elite chilena considera que los medios de comunicación son la institución más influyente del país, tal como se observa en el cuadro II.

\section{Cuadro II: El Poder en Chile ${ }^{15}$}

(Las primeras 5 son las más influyentes y las últimas 5 las menos)

\begin{tabular}{|c|c|c|}
\hline Ránking Poder & Institución & Puntaje \\
\hline 1 & Medios de Comunicación & 8.6 \\
\hline 2 & Ministerios del área Económica & 8.3 \\
\hline 3 & Grandes Grupos Económicos & 8.0 \\
\hline 4 & Banco Central & 7.4 \\
\hline 5 & Ministerios del área Política & 7.2 \\
\hline 28 & Empresas de transporte & 4.4 \\
\hline 29 & Artistas e Intelectuales & 4.2 \\
\hline 30 & Colegios Profesionales & 3.7 \\
\hline 31 & ONGs y Fundaciones & 3.6 \\
\hline 32 & Asociaciones sindicales & 3.4 \\
\hline
\end{tabular}

Fuente: Encuesta Elite, PNUD, 2004.

El cuadro muestra la relevancia de los medios de comunicación en la sociedad moderna y, especialmente, en Chile. Por ello, cualquier estrategia de intervención comunicacional a favor de la igualdad requiere necesariamente de los medios masivos de comunicación, ya sean nacionales, locales o comunitarios. "Los medios actúan de mediadores entre la realidad global y el público o audiencia que se sirve de cada uno de estos medios" $" 16$.

Existen teorías que avalan la influencia de los medios en la sociedad, de hecho, en ellas se han basados programas de seguridad ciudadana, por ejemplo. "Los medios masivos de comunicación tienen la habilidad de difundir y en ocasiones imponer ciertas nociones acerca de la naturaleza de la realidad social. Esta habilidad se deriva de dos instancias: en primer lugar de la uniformidad del sistema de mensajes que refleja los valores, creencias o

\footnotetext{
${ }^{14}$ BRUNNER, José Joaquín, BARROS, Alicia y CATALÁN, Carlos. Transformaciones culturales y modernidad. FLACSO. Santiago de Chile. 1989.

${ }^{15}$ El "Poderómetro" se construyó sobre la base de una encuesta a miembros de la elite chilena. Se propuso a los entrevistados un listado de 32 entidades y se solicitó evaluar cada una de ellas a partir de la pregunta: ¿cuánta influencia cree usted que tienen hoy en Chile cada uno de ellos? Las calificaciones se hicieron en una escala de 1 a 10, donde 1 significa que la entidad calificada no tiene influencia y 10 que tiene mucha influencia. Se identifican las 5 instituciones más influyentes en Chile y las 5 menos influyentes.

${ }^{16}$ GOMIZ, Lorenzo. Teoría del Periodismo. Cómo se forma el presente. Ediciones Paidós. Barcelona. 1991.
} 
comportamientos convencionales tendientes a perpetuar el status quo; y en segundo lugar del realismo con que los medios presentan esa visión uniformada de la realidad social" ${ }^{, 17}$.

La construcción social de la realidad por los medios masivos de comunicación es un proceso de producción, circulación y reconocimiento ${ }^{18}$. Los medios masivos de comunicación constituyen su principal código a partir de la distinción informable/no informable (o noticia/no noticia). A partir de esta distinción, también se genera la opinión pública, que de acuerdo con Luhmann permite la autoobservación de la sociedad, por lo tanto, permite observar la desigualdad como un problema social de múltiples alcances. Según Luhmann, cualquier juicio sobre las posibilidades de la autodescripción de la sociedad moderna se debe ajustar a los medios masivos de comunicación (imprenta, cine, radio, televisión). Y la selectividad de aquello que consideran estos medios se refleja como opinión pública, que se puede describir en tres dimensiones del sentido.

En la dimensión material, los datos cuantitativos adquieren un significado extraordinario (lo que se llama "el lenguaje de los números"), sin que exista la posibilidad de reflexionar cómo se calculan. Por ejemplo, se aceptan sin reparos los datos que indican un aumento del producto interno bruto o se lamenta su caída. La política y la bolsa de comercio reaccionan ante el hecho de que las cifras sean importantes, sin que nadie se moleste en pensar que el PIB puede caer por el simple hecho de que aumenten los accidentes o catástrofes. En la dimensión temporal, lo que merece ser mencionado por los medios de comunicación debe ser nuevo, es decir, tener el carácter de evento o de noticia, o sea, actual y novedoso. Sobre esta base se organiza la profundidad temporal de aquello a lo que se hace referencia. La dimensión social se presenta como conflicto, pero en el fondo existe una expectativa continua de que, finalmente, se debería llegar a un acuerdo. Todos estos filtros reaccionan como un refuerzo de la irritación del sistema. Entonces, en el plano de lo que se comunica y de lo que es generado por la capacidad de producir relaciones en la comunicación, la sociedad aparece como una entidad que se irrita a sí misma, o sea, que se alarma sola ${ }^{19}$.

La desigualdad puede constituirse en la alarma de las economías emergentes y es por ello que se debe enfatizar su disminución para evitar problemas sociales futuros, o para aminorar los riesgos. La posibilidad de generar operaciones que permitan evitar consecuencias desastrosas se denomina prevención en la Teoría de los Sistemas Sociales. "Es una preparación contra daños futuros no seguros, buscando ya sea que la probabilidad de que tengan lugar se disminuya o que las dimensiones del daño se reduzcan. La prevención se puede practicar, entonces, tanto ante el peligro como ante el riesgo" 20 .

$\mathrm{Y}$ es aquí donde surge la posibilidad de una intervención comunicacional como herramienta para la disminución de la desigualdad social. No obstante, es importante rescatar conceptos de la Teoría de la Acción Comunicativa de Habermas, porque aunque el consenso en una sociedad compleja es altamente improbable, sí es posible establecer ciertas semánticas, que condensadas funcionan como un soporte en la sociedad. Es lo que sucede actualmente con la democracia dentro del sistema político occidental, que se estableció

\footnotetext{
${ }^{17}$ DASTRES, Cecilia y MUZZAPAPPA, Eva. La comunicación como estrategia para orientar a la ciudadanía frente a la violencia y criminalidad. Centro de Estudios en Seguridad Ciudadana de la Universidad de Chile. Santiago de Chile. 2003.

${ }^{18}$ ALSINA, Miquel. La construcción de la Noticia. Ediciones Paidós. Barcelona. 1999.

${ }^{19}$ LUHMANN, N. y DEGIORGI, R. Op. Cit.

${ }^{20}$ LUHMANN, Niklas. Sociología del riesgo. Universidad de Guadalajara, Universidad Iberoamericana. México. 1992.
} 
como comunicación desviante, para luego ser seleccionada y reestabilizada como la mejor forma de gobierno, es decir, se estableció como el programa de gobierno en la mayoría de los Estados occidentales. Esta conceptualización se relaciona con la evolución de las sociedades que establece Luhmann.

Este autor propone tres procesos evolutivos: variación, selección y reestabilización. La variación se refiere a la modificación de los elementos del sistema, es decir, las comunicaciones. La variación consiste en una reproducción desviante de los elementos del sistema. O sea, éste se constituye a través de una comunicación inesperada, sorpresiva que altera el estado del sistema.

El segundo proceso, la selección, se refiere a las estructuras del sistema, es decir, a las expectativas que guían a la comunicación. Tomando como base una comunicación desviante, la selección elige las referencias de sentido que prometen tener valor de construcción de una estructura, que pueden producir el efecto de construir y de condensar expectativas. Con la selección luego se rechazan explícitamente las innovaciones que no parezcan idóneas para convertirse en estructura, es decir, que no parezcan idóneas para fungir como directiva de la comunicación ulterior.

El tercer proceso, la reestabilización, consiste en la formación de sistemas a las que algunas innovaciones les aseguran duración y capacidad de resistencia. Aquí se trata del mismo sistema de la sociedad con relación a su entorno. Con la evolución de la sociedad, la función de reestabilización se traslada cada vez más hacia los sistemas parciales de la sociedad, que deben lograr afirmarse en el entorno interno de la sociedad. Por ello, el problema será, en última instancia, la misma posibilidad de mantener la diferenciación de los sistemas de la sociedad ${ }^{21}$.

A partir de los tres procesos evolutivos, se puede concluir que la evolución es la selección de selecciones. Por ello, a través de la comunicación se puede provocar una irritación en la sociedad que permita su evolución hacia una sociedad más igualitaria.

\section{La comunicación de la igualdad}

Pese a que los planteamientos de Luhmann y de Habermas en torno a la comunicación difieren en aspectos centrales, se relacionan en la importancia que le asignan a la comunicación en la construcción de la sociedad. Para Luhmann, la comunicación no tiene una racionalidad, un fin, en cambio, para Habermas ésta permite que los actores sociales den razones a sus actos y acaten las razones de los otros. Y esta acción comunicativa se da en "el mundo de la vida", que es un lugar de la cultura donde los sujetos se entienden y razonan.

Estos conceptos los recoge la siguiente propuesta de intervención comunicacional, que no escapa por cierto a las conceptualizaciones de la sociedad diferencialmente funcional que plantea Luhmann. Es decir, a partir de ambas teorías, se propone una intervención comunicacional que recoge planteamientos también de la antropología social aplicada y de la interculturalidad.

Para llevar a cabo esta tarea de intervención social a partir de la comunicación se requieren de los mismos procedimientos que para cualquier tipo de intervención, principalmente de la antropología. Estos métodos son: estudios previos, planificación,

\footnotetext{
${ }^{21}$ LUHMANN, N. y DEGIORGI, R. Op. Cit.
} 
ejecución y evaluación ${ }^{22}$. Estas técnicas permiten una unión entre el conocimiento científico y la acción práctica.

Por ello, se requiere primero conocer las tipologías que unen a comunicación y desarrollo. Existen algunas conceptualizaciones que han sido sistematizadas por Adalid Contreras $^{23}$ (2000). La primera de ellas hace mención a la "Comunicación del desarrollo", que es un proceso de incorporación de los países en vías de desarrollo dentro del sistema comunicativo mundial para la difusión de la tecnología industrial, las instituciones sociales modernas y el modelo de sociedad de libre mercado, donde el creciente flujo de información sería un elemento importante para configurar un sentimiento nacionalista a favor del desarrollo, y los medios de comunicación de masas ocupan un lugar central, en la medida que se entienden como un subsistema clave para generar el cambio social, mediante la persuasión, educación y aculturación no conflictiva de la población expuesta a mensajes exaltadores del progreso, movilidad social, realización personal, innovaciones, alfabetización y consumismo.

También se considera la conceptualización de "Comunicación de apoyo al desarrollo", donde la comunicación instrumentalizada a las acciones de cambio sufre reduccionismos didactistas en los procesos educativos; reduccionismos tecnologistas en la concepción y manejo de los medios; reduccionismos organicistas en el énfasis en los liderazgos y organización social; y reduccionismos epistemológicos en la exaltación de los mensajes como base para la igualación de emisores y receptores.

Y, por último, se habla de "Comunicación con desarrollo", donde se entiende que la comunicación se enriquece con los estudios de recepción y consumo cultural, diseñando un paradigma que valora las mediaciones y resignificaciones en la apropiación y uso de los mensajes desde la complejidad de las culturas. "Mattelart nos habla del retorno al sujeto, al rol activo del receptor y usuario de los flujos y redes de comunicación; y Martín-Barbero nos va a proponer entender la comunicación en sus mediaciones, desde la densidad de las relaciones cotidianas sin diferencias entre una cultura de masas y otra altruista popularliberadora en la trama espesa de los mestizajes y las deformaciones de lo urbano, de lo masivo" 24 .

A partir de la tipología "Comunicación con desarrollo", esta intervención comunicacional propone una comunicación de la igualdad, con el fin de construir una semántica que establezca a la igualdad como premisa elemental de la sociedad. Principalmente, la igualdad en la distribución de la riqueza, pero también en todos los derechos colectivos e individuales, es decir, en lo referente a la discriminación, la marginalidad, la delincuencia y todos aquellos problemas sociales que tienen como distinción principal el código igual/desigual.

Esta comunicación de la igualdad debe considerar la relevancia de los medios masivos de comunicación, no solo los medios tradicionales, sino también aquellos que están en contacto directo con las comunidades, es decir, los medios locales, regionales, comunitarios o vecinales. Esta comunicación se requiere tanto para las políticas públicas, como proyectos privados empresariales o personales.

\footnotetext{
${ }^{22}$ CADENAS, Hugo. La antropología aplicada en una sociedad compleja. Revista MAD. Departamento de Antropología, Universidad de Chile. Santiago de Chile. 2005.

${ }^{23}$ CONTRERAS, Adalid. Comunicación-desarrollo para 'otro occidente'. Revista Razón y Palabra, № 18. México. 2000.

${ }^{24}$ Ibid.
} 
La comunicación de la igualdad debe adoptar una postura multicultural, que es la relativización valórica que rompe con el etnocentrismo y se abre a un conocimiento tolerante, múltiple y progresivamente construido por la inducción ${ }^{25}$. Es decir, necesariamente esta comunicación debe considerar cada uno de los elementos presentes en la cultura local o nacional para intervenir.

Por ejemplo, si existe una política pública destinada a promover el acceso igualitario a la salud, se puede construir una comunicación que fomente y legitime esta práctica considerando las distintas concepciones de salud y enfermedad presentes en una cultura. Para esto, la labor de un antropólogo puede ser fundamental. Por eso, la intervención comunicacional en una sociedad compleja es un trabajo interdisciplinario, donde se trata de responder a la creciente diferenciación de la sociedad, a su heterogeneidad progresiva.

Otro aspecto necesario en una intervención comunicacional es la distinción entre los sistemas de interacción y los sistemas organizacionales. Los primeros se forman cuando, para resolver a través de la comunicación el problema de la doble contingencia, se usa la presencia de personas. Los presentes se imponen como personas haciéndose ver y escuchar, por eso, fácilmente hacen comprender lo que tienen que hacer en la interacción. A la autorregulación de la interacción contribuye el hecho de los participantes se deben reciproco respeto y pueden esperar un respeto reciproco de los roles que cada uno tiene fuera de la interacción ${ }^{26}$.

Tal como la sociedad y la interacción, la organización es una forma determinada por la relación de doble contingencia. Esto, porque cada una de las personas puede reaccionar de manera diferente y puede rechazar o aceptar los deseos o expectativas del otro. Pero al entrar en una organización se dan por aceptados algunos vínculos y se constituye así la cualidad de miembro de la organización.

El pertenecer a una organización se apoya en la movilidad, y la movilidad debe ser admitida socialmente. Por ello, la solución de la doble contingencia consiste en que la pertenencia a la organización puede estar condicionada por una premisa que permite mantener el status. Las organizaciones se comunican preferentemente con organizaciones y son las organizaciones las que pueden armonizar entre ellas enormes cantidades de interacciones. Cuando las organizaciones operan en sistemas de funciones deben ser consideradas como sistemas sociales operacionalmente clausurados, independientes, basados en su ámbito de decisiones, ya que las decisiones sólo se pueden tomar y ejecutar en las organizaciones mismas ${ }^{27}$.

Entonces, las redes comunicacionales pueden tejerse de una manera interaccional (cara a cara, puerta a puerta) o organizacional (municipios, juntas de vecinos, medios de comunicación. Esta distinción permite estructurar estrategias de comunicación desviante que promueven el cambio social a favor de la igualdad, para luego ser seleccionadas por los sistemas parciales (económicos, políticos, entre otros) que generan las estructuras más idóneas para justamente propiciar una mayor igualdad (redistribución de la riqueza, reforma tributaria, leyes de protección a la diversidad). Finalmente, estas estructuras son reestabilizadas en la sociedad y se acepta la comunicación de la igualdad como una semántica condensada.

\footnotetext{
${ }^{25}$ WEIBEL, Mauricio. Comunicación intercultural y gestión pública. Desafíos de participación y gobernabilidad. Instituto de la Comunicación e Imagen, Universidad de Chile. Santiago de Chile. 2005.

${ }^{26}$ LUHMANN, N. y DEGIORGI, R. Op, Cit.

${ }^{27}$ Ibid.
} 
En la perspectiva de Habermas, la acción comunicativa, entonces, permite en este caso que la igualdad sea el consenso en la sociedad y que las acciones tanto en el mundo de la vida como en el sistema de la sociedad estén orientadas por esta racionalidad. Este acuerdo comunicativo, siguiendo con el optimismo de este pensador alemán, permite un nivel de razonamiento y entendimiento comunicativo que posibilita la integración social.

Justamente, la desigualdad promueve lo contrario, es decir, la desintegración de lo social, la diferencia entre excluidos e incluidos. Por ello, la intervención comunicacional puede ser una herramienta más para prevenir los riesgos de una sociedad que tiende la concentración de la riqueza y a la marginación de los sectores más vastos de la población, como ocurre actualmente en Chile. 